\title{
The Use of Synchrotron Radiation as an Absolute Source of VUV Radiation
}

\author{
D. L. Ederer, E. B. Saloman, S. C. Ebner, and R. P. Madden \\ Institute for Basic Standards, National Bureau of Standards, Washington, D.C. 20234
}

(September 10, 1975)

\begin{abstract}
Synchrotron radiation has been used as a standard source to calibrate spectrographic instruments at the National Bureau of Standards (NBS). Conceptually it is straightforward to apply the calculable continuum distribution of synchrotron radiation to problems requiring a source of known irradiance if the electron energy, the radius of the electron orbit, and the beam current are known. In practice many factors affect the accuracy of such a calibration, such as temporal and spatial variations in the electron beam, uncertainties in the orbital radius and maximum energy of the orbiting electron beam. These sources of error are discussed and the method of calibration on SURF-I is specified. A storage ring synchrotron radiation facility (SURF-II) is now operational at NBS. The calibration techniques developed for SURF-I are applied to SURF-II with anticipated improvements in calibration accuracy. For SURF-I the incident flux was determined with an accuracy of 15 percent while for SURF-II we anticipate accuracies of about 7 percent.
\end{abstract}

Key words: Irradiance; radiometry; spectrometer calibration; standard source; synchrotron radiation; vacuum ultraviolet.

\section{Introduction}

The need for vacuum ultraviolet (vuv) source and detector standards has been growing steadily during the past decade with the requirements of space physics, aeronomy, and plasma physics setting the pace. Reliable detector transfer standards have been developed $[1,2]^{1}$ for use over a wavelength range from 200 to $2500 \AA$. While detector-transfer-standard systems cover a broad spectral range in the vuv, source standards are not as well developed.

The simplest calibration method is the substitution of a calibrated standard for the unknown in an existing system. This makes source standards the standards of choice in calibrating other sources. The use of detector standards for this purpose requires independent knowledge of the monochromator optical system efficiency. On the other hand, detector standards are the standards of choice for calibrating other detectors.

Several sources have been devised [3-15] for use as standards in the vuv, however the 1000 to $3000 \AA$ spectral range is where the most intense development has taken place. Among the various proposed sources synchrotron radiation [3-6] has appeared especially attractive. In addition, the radiation from wall-stabilized arcs $[7,8]$, transition radiation $[9,10]$, and discharge

${ }^{1}$ Figures in brackets indicate the literature references at the end of this paper. lamps [11, 12] have also been demonstrated as feasible sources which can be characterized sufficiently well to be used as standards. Branching ratio techniques [13-15] have also proven useful in certain situations.

The characteristics of these different sources are given in table 1 for comparison. It is clear that the features pertaining to each source serve to specialize its use. The best choice must be made with a given experiment in mind. For example, while both the synchrotron and wall-stabilized arc are possible standard sources, one would be drawn to the use of a synchrotron at wavelengths less than $1200 \mathrm{~A}$, the lithium fluoride cutoff, simply because an expensive differential pumping system is unnecessary. On the other hand if a filled optical system is a more important criterion than high intensity, one would use a transition radiation source which in addition to its greater divergence is also more portable than either a synchrotron or a wallstabilized arc. While for the 1600 to $2600 \AA$ spectral range a deuterium lamp [12] is the simplest to use. All the sources mentioned except the synchrotron have cutoffs above $500 \AA$. At wavelengths less than $500 \AA$ the synchrotron remains the only effective candidate for a continuum calibration source.

Wall-stabilized arcs and sources of synchrotron radiation are considered absolute sources since their radiation is a known function of their operating parameters. Both, unfortunately, are rather immobile and require some means of transferring the calibration to 
TABLE 1. Characteristics of vuv standard sources

\begin{tabular}{|c|c|c|c|c|c|c|}
\hline Source & $\begin{array}{l}\text { Synchrotron } \\
\text { radiation }\end{array}$ & $\begin{array}{l}\text { Wall-stabilized } \\
\text { arc }\end{array}$ & $\begin{array}{l}\text { Transition } \\
\text { radiation }\end{array}$ & $\begin{array}{l}\text { Cerenkov } \\
\text { radiation }\end{array}$ & $\begin{array}{l}\text { Branching ratio } \\
\text { technique }\end{array}$ & $\begin{array}{c}\text { Discharge and } \\
\text { deuterium lamps }\end{array}$ \\
\hline $\begin{array}{l}\text { Relative }(R) \text { or } \\
\text { absolute }(\mathrm{A}) \\
\text { standard }\end{array}$ & $A$ & $A^{\mathrm{b}}, R$ & $A$ & $A$ & $A$ & $R$ \\
\hline $\begin{array}{l}\text { Line }(L) \text { or con- } \\
\text { tinuous }(\mathrm{C}) \\
\text { source }\end{array}$ & $C^{\mathrm{a}}$ & $C$ & $C$ & C & $L$ & $L, C$ \\
\hline $\begin{array}{l}\text { Wavelength range } \\
(\AA)\end{array}$ & $>0.1$ & $1300-3600$ & $>500$ & $>1200$ & $900-3000^{c}$ & $>304^{\mathrm{d}}$ \\
\hline Maximum intensity & Bright $^{\mathrm{f}}$ & Bright & $\operatorname{Dim}^{\mathrm{g}}$ & Dim & Intermediate & Intermediate \\
\hline $\begin{array}{l}\text { Scalable in } \\
\text { intensity? }\end{array}$ & Yes & Limited $^{\mathrm{e}}$ & Yes & Yes & Limited & Limited \\
\hline$f$ Number & Small $(f / 500)$ & Small & Large $(f / 10)$ & Large & Large & Large \\
\hline Polarized? & Yes & No & Yes & Yes & No & No \\
\hline $\begin{array}{l}\text { Vacuum in } \\
\text { source }\end{array}$ & High & Poor & High & High & Poor & Poor \\
\hline Physical size & Massive & Large & Small & Small & Small & Small \\
\hline
\end{tabular}

Notes:

aAdjustable wavelength distribution; storage ring very stable.

${ }^{\mathrm{b}}$ Only hydrogen arc is absolute.

'Usable only at selected wavelengths; subject to absorption effects; portable.

the unknown source. Transition radiation sources and the deuterium lamp have been suggested $[9,10,12]$ for this purpose in the vuv.

Transferring a calibration from a standard source in one laboratory to an unknown source in a second laboratory effectively involves the calibration of two spectrometers, one at the laboratory housing absolutetransfer standards and the other in the field where the transfer standard is compared with the unknown source. There are significant advantages in an alternate approach; namely to directly calibrate the spectrometer that is to be used for determining the radiance of the unknown source. If the calibration is made on a before-after basis, any changes in sensitivity can be interpolated, and errors resulting from a double transfer are eliminated. This method requires direct illumination (no auxiliary mirrors) of the spectrometer by both standard and unknown sources. When NASA encouraged us to establish a source calibration facility at the National Bureau of Standards "Synchrotron Ultraviolet Radiation Facility" (NBS-SURF) for the Skylab program we took the approach outlined above, namely, calibrate a spectrometer to be used as a transfer standard. We used synchrotron radiation as the absolute standard source because the required calibration range, 200 to $1200 \AA$, was beyond the range of all other sources. The use of synchrotron radiation to calibrate a spectrometer above $500 \AA$ has recently been reported [16].

The basic assumption of our calibration method is that the intensity distribution of synchrotron radiation is a calculable quantity. Once this assumption is granted, it is straightforward conceptually to apply the

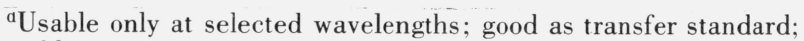
portable.

e Over factor of ten.

${ }^{\mathrm{f}}$ A bout $10^{16}$ photons s $\mathrm{A} \cdot \mathrm{Sr}$.

${ }^{g}$ About $10^{10}$ photons s $\AA \cdot \mathrm{Sr}$.

calculable continuum distribution of synchrotron radiation to problems requiring a source of known irradiance provided the electron energy, the radius of the electron orbit, and the beam current are known. In practice many factors affect the accuracy of such a calibration and this paper will discuss the steps taken at NBS to attain a successful calibration. In the following sections the measurements required to determine the parameters necessary for the calibration will be presented. Systematic errors, their sources and cure, will be a topic of discussion, as will some special problems that arise due to the peculiar characteristics of the radiation source. While this paper will deal primarily with synchrotoron radiation that arises from the periodic acceleration of electrons in a synchrotron, some time will be devoted to the application of synchrotron radiation from a storage ring to spectrometer calibrations because the NBS synchrotron (SURF-I) has now been converted into an electron storage ring (SURF-II).

\section{The Synchrotron as a Primary Radiation Standard}

We must first discuss the validity of the fundamental assumption involved in using synchrotron radiation as an absolute source standard: that the photon flux distribution in both wavelength and angle is an exactly calculable quantity. In the following paragraphs ample evidence has been given to justify this basic assumption. The reader is referred to several excellent reviews [17-19] of the historic development of the use of synchrotron radiation for a more detailed account of 
the characteristics of the radiation and for other applications of it.

In their classic paper Tomboulian and Hartman [3] suggested that synchrotron radiation could be used as an absolute radiation standard because of the calculability of the radiant energy wavelength distribution. This suggestion arose as a result of their experiments that compared measured synchrotron radiation intensity with the calculated intensity distribution. Their experiments covered different wavelength regions, from the quartz ultraviolet to the vacuum ultraviolet, as well as both radiation from monoenergetic electrons and electrons accelerated over a full machine cycle. They measured the angular distribution of the radiation at several wavelengths and electron energies. The result of this extensive study demonstrated the classical theory was appropriate to the description of synchrotron radiation.

Nine years later Codling and Madden [4] reported measurements of the polarization properties of the radiation in the visible and also used radiometric techniques to measure the beam current. A year later a group [5] at DESY (Deutsches Elektronen-Synchrotron) compared the relative intensity distribution as a function of photon energy and azimuthal angle relative to the orbital plane. In this case the electron energy varied between $4 \mathrm{GeV}$ and $6 \mathrm{GeV}$ and the photon energy extended between $20 \mathrm{keV}$ and $300 \mathrm{keV}$. Their results substantially confirmed the measurements of Tomboulian and Hartman [3] at lower electron and photon energies, namely, that the measurements were in agreement with calculations to within the experimental error of order 10 percent.

Lemke and Labs [6], working at DESY, explored the possibility of using synchrotron radiation as a fundamental radiometric standard in the wavelength region 2200 to $5500 \AA$. They compared the spectral radiance from the synchrotron with that of a standard lamp. From this comparison they were able to measure the relative spectral distribution for their geometry as well as independently determining the circulating electron current. Applying the appropriate polarization correction (synchrotron radiation is highly polarized in the orbital plane) they obtained a relative spectral distribution that agreed with the computed flux within 2 percent. Their optical measurement of the circulating current was consistent with an independent direct measurement of the beam current to within the 15 percent limit of experimental error of this direct technique. These experiments have shown the measurements to be consistent with the calculations of the flux over a broad spectra range for accelerated electrons over a wide energy range. At the Glasgow University synchrotron, Key [20] made a very careful relative flux determination between $3500 \AA$ and $6000 \AA$ by comparing the radiance from the electron beam with the radiance from a standard lamp. This comparison yielded coincidence to $\pm 1 / 2$ percent between the relative measured spectral distribution and the predicted spectral distribution.

In $1969 \mathrm{Pitz}$ [11] used synchrotron radiation as a primary standard to calibrate mercury, xenon, and deuterium transfer standard lamps in the wavelength range 1500 to $2700 \AA$. Later a mercury lamp and a deuterium lamp traceable to one calibrated by Pitz was compared to a wall-stabilized hydrogen arc [21]. Discrepancies were found to be about 10 percent between $1650 \AA$ and $2550 \AA$. Between $2550 \AA$ and $2700 \AA$, the long wavelength limit of the calibration, the deviations were as great as 25 percent. Whether these discrepancies were due to aging of the lamp or some other source is not clear, however evidence points to a lamp change rather than something faulty with the synchrotron radiation flux determination. More recently a comparison [22] between a wallstabilized arc and synchrotron radiation has been made through the use of deuterium lamp and xenon lamp transfer standards at a wavelength near $1650 \AA$. The resulting calibrations agreed to \pm 20 percent at low arc currents and \pm 5 percent at the higher currents. The authors concluded that the discrepancies were consistent with errors expected from the synchrotron calibration and the wall-stabilized arc calibration.

From these measurements the validity of the fundamental assumption underlying the use of synchrotron radiation as an absolute standard seems to be well founded. Not only have direct measurements of the spectral distribution confirmed the calculable nature of synchrotron radiation but independent calibrations of transfer standards with synchrotron radiation and with the wall-stabilized arc have also been found consistent.

\section{Method}

It is conceptually straightforward to apply the calculable continuum distribution of synchrotron radiation to spectrometer calibration since the efficiency, $\epsilon(\lambda)$, of the spectrometer at wavelength $\lambda$. is given by:

$$
\epsilon(\lambda)=\frac{F(\lambda, \Delta \Omega)}{R(\lambda, \Delta \Omega)},
$$

where $F(\lambda, \Delta \Omega)$ is the flux incident on the entrance aperture of the spectrometer which subtends a solid angle $\Delta \Omega$ located at some angle $\theta$ with respect to the orbital plane, and $R(\lambda, \Delta \Omega)$ is the response of the instrument to the incident flux. In order to understand the problems involved in the use of synchrotron radiation and to compute the quantity $F(\lambda, \Delta \Omega)$ it is necessary to delve into the properties of the radiant flux emitted by this source.

\subsection{Properties of Synchrotron Radiation}

The radiation emanating from centripetally accelerated electrons is confined along the direction of motion due to relativistic effects. The average divergence angle of the radiation is $m_{0} c^{2} / E$ where $m_{0}$ is the electron rest mass and $E$ is the instantaneous energy of the electrons. Since $E \gg m_{0} c^{2}$, the radiation from most 
machines is confined to an angle of a few milliradians above and below the orbital plane. Because of the electron orbital motion the radiation is uniform in the orbital plane and at a given energy and radius is a function only of the azimuthal angle. A schematic view of the radiation pattern is shown in figure 1 .

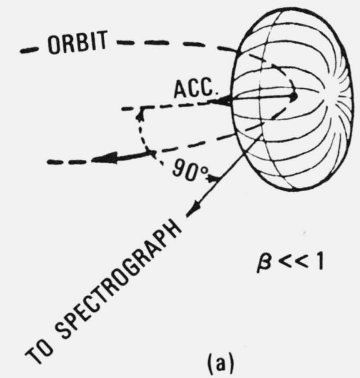

(a)

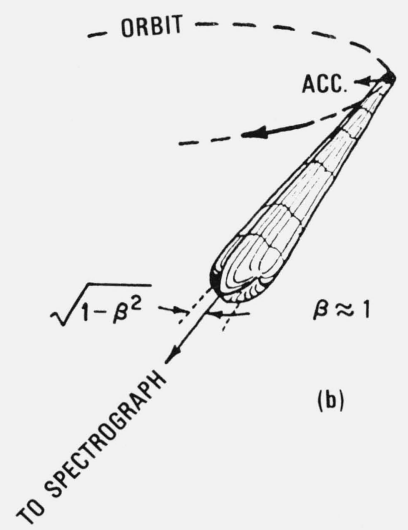

FIGURE 1. Angular radiation patterns for orbiting electrons of greatly different energies.

(a) Nonrelativistic energy, showing the typical dipole pattern; (b) Very relativistic energies, showing that the dipole pattern has been greatly warped around to the forward direction of the electron (from ref. [3]).

This rather narrow distribution presents the first problem for spectrometer calibration since most other sources, whose radiance is to be determined through the use of the calibrated spectrometer, have a much different angular distribution. In addition the radiation from the synchrotron source will often not fill the entrance solid angle of the instrument.

The power radiated by monoenergetic, accelerated electrons is a continuum which has the form $[23,3]$

$$
\begin{aligned}
P(E, R, \theta, \lambda)=\frac{4 e^{2} c R}{3 \lambda^{4}} \gamma^{-4}\left(1+X^{2}\right)^{2} & \\
& {\left[K_{2 / 3}^{2}(\xi)+\frac{X^{2}}{1+X^{2}} K_{1 / 3}^{2}(\xi)\right] }
\end{aligned}
$$

where $P(E, R, \theta, \lambda)$ is the power in ergs per second per radian per electron per angstrom, $\gamma=E / m_{0} c^{2}$, and $\xi=(2 \pi R / 3 \lambda) \gamma^{-3}\left(1+X^{2}\right)^{3 / 2}$ with $X=\gamma \theta$. The quantity $R$ is the orbital radius and $\theta$ is the azimuthal angle with respect to the orbital plane while $e$ and $c$ are the electron's charge and the speed of light respectively. The quantities $K_{2 / 3}(\xi)$ and $K_{1 / 3}(\xi)$ are modified Bessel functions of the second kind. This the fundamental equation that is used to compute the flux incident upon the entrance aperture of a spectrometer. For a storage ring where the instantaneous energy $E$ is a constant it is only necessary to integrate eq (2) over the entrance aperture solid angle to obtain the flux per electron at wavelength $\lambda$. However, for many accelerators (of which SURF-I, the old NBS-180 MeV synchrotron was an example) the energy of the electrons varies as they are periodically accelerated from zero to maximum energy. In this case eq (2) must be integrated over the time variation of the electron energy as well. Figure 2 shows the angular

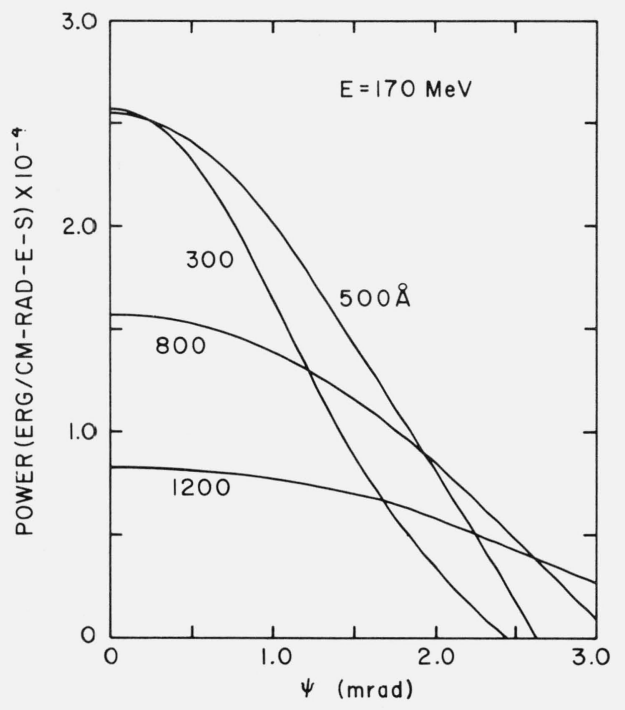

FIGURE 2. Angular distribution of synchrotron radiation at several wavelengths for accelerated electrons with a maximum energy of $170 \mathrm{MeV}$.

variation of the flux for accelerated electrons at several wavelengths. Equation (2) was integrated numerically for a time variation of the electron energy given by $E(t)=E_{\max } \sin \omega t$. Typical operating conditions for the NBS synchrotron were chosen, namely $E_{\max }=170 \mathrm{Mev}$, $R=83.4 \mathrm{~cm}$, and $\omega=2 \pi / T=2 \pi 60 \mathrm{~s}^{-1}$. The ordinate defines the power $P\left(E_{\max }, \theta, \lambda\right)$ in ergs per second per centimeter per radian of azimuthal angle per electron. The quantity $P\left(E_{\max }, \theta, \lambda\right)$ is given by

$$
P\left(E_{\max } \theta, \lambda\right)=\frac{1}{T} \int_{E\left(t_{1}\right)}^{E\left(t_{2}\right)} P(E, R, \theta, \lambda) d E(t) .
$$

The limits of integration $E\left(t_{1}\right)$ and $E\left(t_{2}\right)$ are the instantaneous electron energies at $t_{1}$ (chosen to be zero, the beginning of the acceleration cycle) and $t_{2}=5.57 \mathrm{~ms}$ the time when the radio frequency accelerating voltage was turned off. It may be seen from figure 2 that in the wavelength region between $100 \AA$ and $2000 \AA$ the angle at which the flux is smaller by a factor of two is typically a few milliradians.

The power $P\left(E_{\max }, \theta, \lambda\right)$ given by equation (3) was numerically integrated over a round spectrometer aperture $\left(0.836 \mathrm{~cm}^{2}\right.$ area) located $13.26 \mathrm{~m}$ from the tangent point of the orbiting electrons. This was the size of the aperture used for the calibration of a Wadsworth spectrometer. The flux $P\left(E_{\max }, \lambda\right)$ in photons/sec- $\AA-e$ passing through this aperture for two values of the maximum electron energy, $E_{\max }$, is shown in figure 3 . Notice that the flux is almost constant between $400 \AA$ and $1000 \AA$ for $E_{\max }$ equal to $170 \mathrm{MeV}$ (curve A) while the magnitude of the flux at these wavelengths is greatly reduced for $140 \mathrm{MeV}$ 


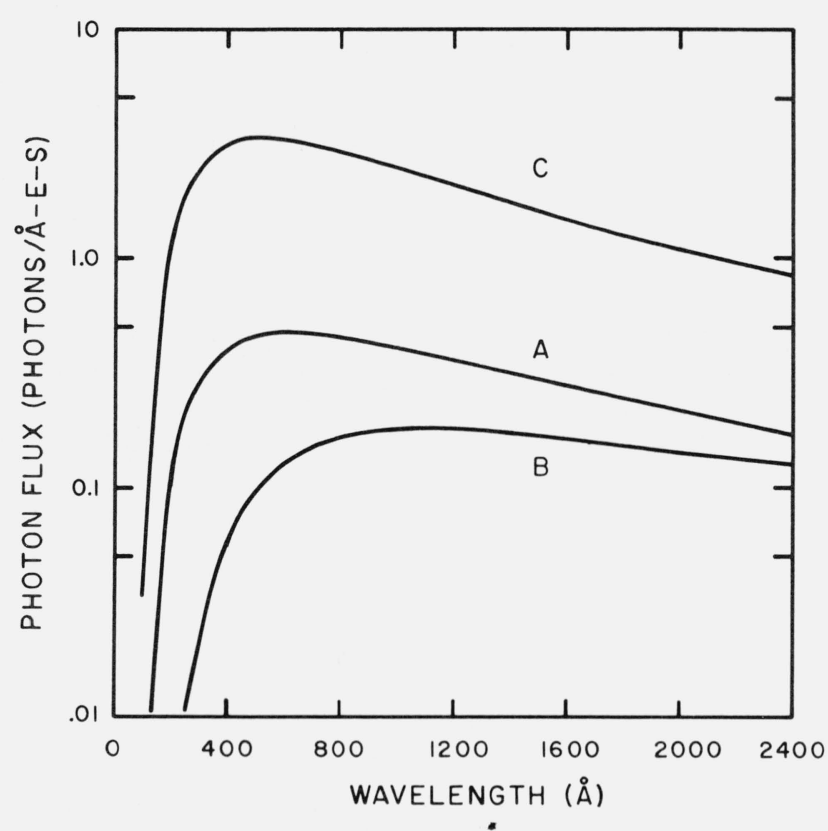

FIGURE 3. Flux passing through a round aperature of solid angle equal to $4.8 \times 10^{7}$ strad as a function of wavelength.

Curve A: Accelerated electron whose energy equals $170 \mathrm{MeV}$. Curve B: Accelerated electrons whose maximum energy is $140 \mathrm{MeV}$. Curve C: Monoenergetic electrons of energy $170 \mathrm{MeV}$.

electrons (curve B). In fact, this great variation in the flux can be used to determine the instrument's response to second order radiation [24]. Curve C in figure 3 is the flux for monoenergetic electrons of 170 $\mathrm{MeV}$ energy obtained by just integrating equation (2) over the aperture. Notice that for the monoenergetic electrons the peak intensity in the spectral distribution is shifted to shorter wavelengths and the flux per electron is significantly greater than for accelerated electrons of the same peak energy averaged over the acceleration cycle. This difference is due both to the duty cycle of the cyclic accelerator and to the fact that the electrons, emit most of their radiation when their instantaneous energy $E(t)$ is nearly equal to $E_{\text {max }}$. The contrast between the two curves A and C emphasizes one of the advantages of using a storage ring for a calibration, namely, for the same number of electrons in the beam, the ring will emit about seven times more radiation than the synchrotron.

In figure 4 we show a comparison between accelerated $170 \mathrm{MeV}$ electrons at an instantaneous current of $5 \mathrm{~mA}$ (typical of SURF-I operation) and $240 \mathrm{MeV}$ monoenergetic electrons at a current of $10 \mathrm{~mA}$ (expected for initial SURF-II operation). Higher flux and the availability of shorter wavelengths make SURF-II a superior calibration source. In addition temporal variation in the radiation from the storage ring is a slowly varying monotonically decreasing function, due to the fact the electron beam lifetime is of the order of an hour, rather than the few milliseconds obtained in a sinusoidally run synchrotron. The fluctuations in the radiation due to variations in the injected current occurs on the order of hours, rather than every few

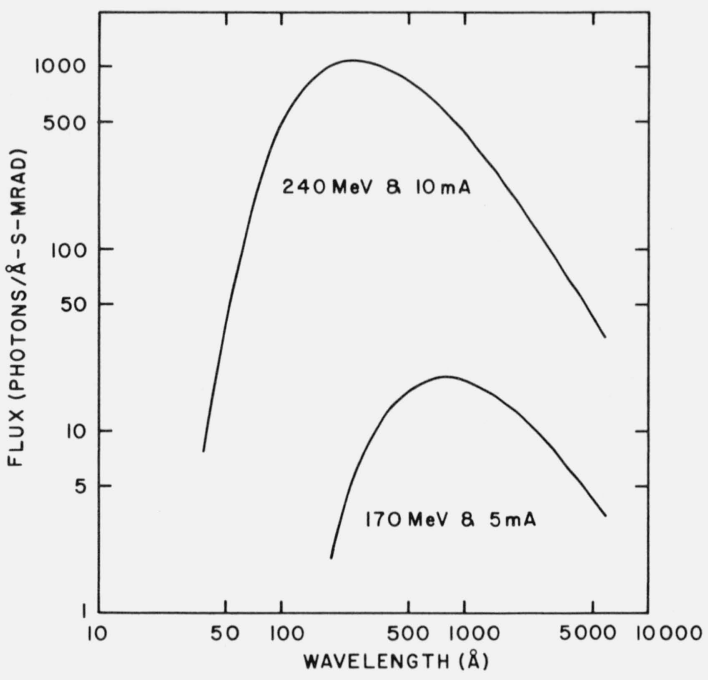

FIGURE 4. Photon flux versus wavelength for (A) a monoenergetic $240 \mathrm{MeV}, 10 \mathrm{ma}$ beam of electrons-typical performance expected for SURF-II and (B) an accelerated $5 \mathrm{ma}$ beam of $170 \mathrm{MeV}$ maximum energy electrons-typical performance of SURF-I.

Multiply ordinate by $1.1 \times 10^{8}$.

milliseconds. These are powerful arguments for the use of a storage ring in spectrometer calibration. Other arguments favoring storage rings will be discussed later.

If the entrance aperture is large enou h to accept the full vertical angular distribution of the radiation, eq (2) can be integrated over the azimuthal angle $\theta$ to yield the flux distribution for monoenergetic electrons:

$$
P(E, \lambda)=\frac{3^{5 / 2}}{16 \pi^{2}} \frac{e^{2} c}{R^{3}} \gamma^{7} G(y)
$$

Similarly for electrons whose energy is given by $E(t)=E_{\max } \sin (\omega t)$ eq (2) can be integrated over angle and averaged over one fourth the energy cycle to yield [3]:

$$
P\left(E_{\max }, \lambda\right)=\frac{3^{5 / 2}}{8 \pi^{3}} \frac{e^{2} c}{R^{3}} \gamma^{7} L(y) .
$$

In eq (4) and eq (5) the quantities $G(y)$ and $L(y)$ are universal scaling functions independent of the electron energy and orbital radius. The quantity $y=\lambda_{c / \lambda}$. The critical wavelength $\lambda_{c}$ is given in the following equation:

$$
\lambda_{c}=\frac{4}{3} \pi R \gamma^{-3},
$$

These two functions are plotted in figure 5. The simple functional form of eq (4) and eq (5) are conveniently used with the figure 4 to estimate (since the vertical acceptance angle of the experiment is initially unknown) the flux available to an experimental ap- 


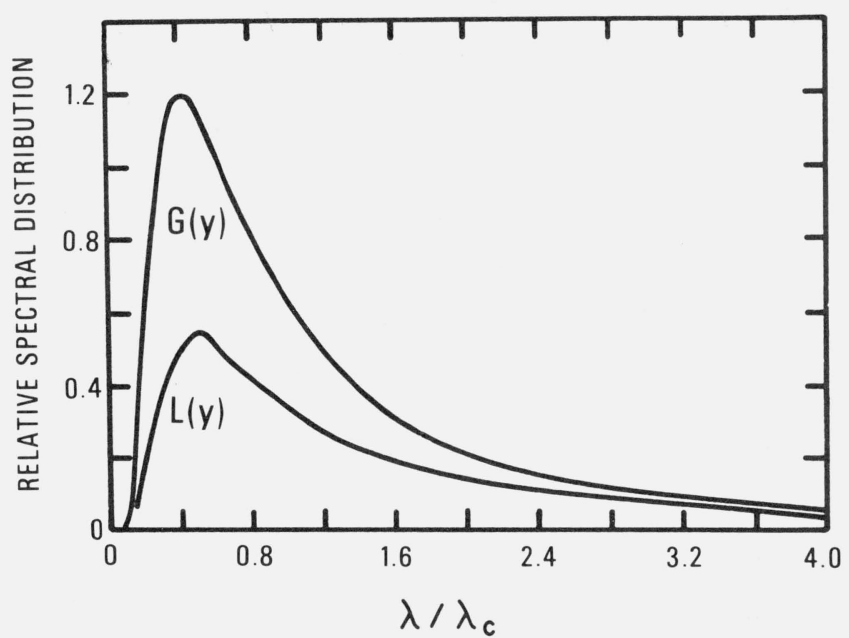

FigURE 5. Universal photon distribution functions for the total angular flux distribution.

$G(y)$, the distribution function for monoenergetic electrons, and $L(y)$, the distribution function for accelerated electrons. The dimensionless quantity $y$ defining the abscissa scale is equal to $\lambda / \lambda c$, where $\lambda c$ is defined in the text.

paratus from a specific machine. A rather tedious computation and integration is thus reduced to a "back of the envelope" calculation.

\subsection{Determination of the vuv Irradiance}

The vuv irradiance $F(\lambda, \Delta \Omega)$ incident on the spectrometer is given by:

$$
F(\lambda, \Delta \Omega)=n_{e} \int_{\Delta \Omega} P\left(E_{\max }, \theta, \lambda\right) d \Omega
$$

where $F(\lambda, \Delta \Omega)$ is the number of photons per $\AA$-s passing through the spectrometer aperture subtending a solid angle $\Delta \Omega$ measured from the tangent point. The quantity $P\left(E_{\max }, R, \theta, \lambda\right)$ was described in the preceeding paragraphs for both monoenergetic and periodically accelerated electrons. $P(E, \theta, \lambda)$ depends on the experimentally determined quantities $R$, the radius of the electron orbit and, $E_{\max }$, the maximum electron energy, in a straightforward way. The quantity $n_{e}$ may be measured by using an induction technique [6] or it can be determined from the synchrotron radiation [4] itself. A particularly elegant technique [25] may be used in the case of photomultiplier calibrations using a storage ring. A reasonably small number of electrons (about 100) is allowed to circulate in the ring. As the electrons are lost from orbit one by one, the decrease in visible emission due to the loss of one electron is noted and from this the exact total number of electrons may be readily obtained. Note with this method the absolute amount of radiation need not be measured. This technique will be available for use at SURF-II. In another method, the radiation is allowed to fall on a suitable calibrated detector. The number of electrons is determined by dividing the measured flux by the flux of synchrotron radiation a single electron radiates to the detector. At SURF-I the spectral interval used for absolute measurement of the synchrotron flux was a $100 \AA$ wavelength band around $5150 \AA$ because an irradiance standard could be used to calibrate a silicon photodiode-filter combination in this band. The visible region of the spectrum was also chosen because for $\lambda>>\lambda_{c}$ (for $170 \mathrm{MeV}$ electrons accelerated at SURF-I $\lambda_{c}=940 \AA$ ) the radiated power is approximately independent of the electron energy and proportional to $\lambda^{-7 / 3}$. Thus small fluctuations in the energy have a negligibly small effect on the determination of the number of electrons in orbit. Thus the number of electrons is given simply by

$$
n_{e}=\frac{F\left(\lambda_{0}, \Delta \Omega\right)}{P\left(E, \lambda_{0}\right)} .
$$

Explicitly writing the integrations over the variation of the detector sensitivity $R(\lambda)$, the filter transmission $T\left(\lambda-\lambda_{0}\right)$ and the radiated power from the synchrotron we have

$$
\begin{array}{r}
F\left(\lambda_{0}, \Delta \Omega\right)=\frac{n_{e}}{2 \pi} \int_{\Delta \Omega} \int_{\lambda_{1}}^{\lambda_{2}} \int_{E\left(t_{1}\right)}^{E\left(t_{2}\right)} \\
P(E, R, \theta, \lambda) T\left(\lambda-\lambda_{0}\right) R(\lambda) d t d \lambda d \Omega
\end{array}
$$

which reduces to

$$
\begin{aligned}
F\left(\lambda_{0}, \Delta \Omega\right) & =n_{e} P\left(E_{\max }, \lambda_{0}\right) \frac{\Delta \Omega}{2 \pi} A \\
& =K V_{F}
\end{aligned}
$$

under the following simplifying assumptions: (a) that $P(E, R, \theta, \lambda) \approx P(E, R, \lambda)$ (the variation of $P(E, R$, $\theta, \lambda)$ over the aperture is about 0.1 percent over a $1 \mathrm{~cm}$ diameter aperture 13.26 meters from the tangent point); (b) the gate time used for determining $F\left(\lambda_{0}\right.$, $\Delta \Omega)$ is short enough so that $P(E, R, \theta, \lambda) \approx P\left(E_{\max }\right.$, $R, \theta, \lambda)$ and $(c)$ the filter is sharp enough so that $P\left(E_{\max }, \lambda\right)=P\left(E_{\max }, \lambda_{0}\right) \quad\left(1+a\left(\lambda-\lambda_{0}\right)\right)$. Under these assumptions the quantity $A$ is a simple calculable factor while $K$ is the detector sensitivity where $F$ watts input to the detector-filter amplifier combination produces a response of $V_{F}$ volts. The quantity $K$ is determined by the detector system response to an irradiance standard. Thus

$$
K V_{S}=\int_{\lambda_{1}}^{\lambda_{2}} \int_{\Delta \Omega^{\prime}} S(\lambda, \theta) T\left(\lambda-\lambda_{0}\right) d \Omega d \lambda
$$

which reduces to

$$
K V_{S}=S\left(\lambda_{0}\right) \Delta \Omega^{\prime} B
$$

if the standard spectral irradiance $S(\lambda, \theta)$ is constant over the solid angle $\Delta \Omega^{\prime}$ and if the filter has a narrow enough bandpass so that $S(\lambda, \theta)=S\left(\lambda_{0}\right)(1+b(\lambda-$ $\left.\left.\lambda_{0}\right)\right)$. The quantity $B$ is eq (11) a calculable factor like $A$ in eq (9). Substituting eq (11) into eq (9) we have 


$$
n_{e}=C \frac{S\left(\lambda_{0}\right)}{P\left(E_{\max }, \lambda_{0}\right)} \frac{V_{F}}{V_{S}}
$$

with $C=2 \pi B \Delta \Omega^{\prime} /(A \Delta \Omega)$ a calculable quantity containing the solid angle factors and the results of integrating eq (8) and eq (10) under the assumptions listed in the preceeding paragraphs.

This approach is valid for both accelerated and monoenergetic electrons. Although the case of an accelerated beam was used as an example, the form of eq (12) is the same for monoenergetic electrons. (The most important difference is that for accelerated electrons one must measure the instantaneous number of electrons since the power averaged over one acceleration cycle is used to compute the flux incident on the spectrometer.) The determination of the number of circulating electrons by this method is essentially reduced to a problem of measuring a voltage ratio. The sources of error intrinsic to this method will be discussed in later paragraphs.

The vuv irradiance incident on the spectrometer can be given in terms of eq (6) and eq (12):

$$
F(\lambda, \Delta \Omega)=C \frac{S\left(\lambda_{0}\right)}{V_{S}} \frac{V_{F}}{P\left(E_{\max }, \lambda_{0}\right)} P\left(E_{\max }, \lambda\right) .
$$

The spectral irradiance incident on the spectrometer is known in terms of calculated quantities and a measured voltage ratio.

Calculations evaluating $P\left(E_{\max }, \lambda\right)$ were carried out to a accuracy of better than 1 percent. For monoenergetic electrons, because the integration over the time variation of the electron energy is eliminated, the accuracy of the calculation is an order of magnitude greater than the uncertainty in the flux due to uncertainties in the electron energy. Determining the response of the instrument, $R(\lambda, \Delta \Omega)$, is the next task.

\subsection{Positioning of Spectrometer}

To determine the flux on the spectrometer, the spectrometer must be aligned at a known angle with respect to the orbital plane of the radiating electrons. In addition, any temporal variation of the inclination of the orbit must be determined since the irradiance is a strong function of the azimuthal angle $\theta$.

Two types of spectrometers have been calibrated at SURF-I: A grazing incidence monochromator [26] and a Wadsworth [27] spectrometer using photographic detection. The instrument to be calibrated, $S$, (see fig. 6) was installed behind the calibrated detector, $C$, aperture assemble, $A$, and an alignment procedure was undertaken with the aid of the visible radiation from the orbiting electrons. The visible radiation was also used to check the effectiveness of the baffling system in eliminating reflections. Since the light beam is almost parallel (average divergence is $3 \mathrm{mrad}$ ) and about $1 \mathrm{~cm}$ in diameter it is impossible to fill the grating of many spectrometers. Therefore, provisions were made to scan the beam over the grating by moving the instrument. It is not necessary to make absolute flux measurements during these scans but only measurements relative to the point of calibration on the grating. For this procedure synchrotron radiation was used for convenience rather than out of necessity. At the storage ring radiation facility, SURF-II, two ports with a direct view of the beam will be provided for instrument calibration. However, at the present time the user must supply his own test chamber to house the instrument to be calibrated.

Synchrotron radiation is highly polarized with the most intense component of polarization lying in the orbital plane. To test the instrument response to this polarization, provisions were made to rotate each instrument about the grating pole. For the grazing incidence monochromator the axis of rotation was coincident with a line drawn from the grating pole to the center of the entrance slit. For the Wadsworth mounting the instrument rotated about an axis passing through the grating pole perpendicular to the rulings. To preserve the geometry of illumination under rotation it was important to align the axis of rotation with the axis of symmetry of the beam. By using the visible radiation from the synchrotron it was possible to make these adjustments without evacuating the instrument chamber. Therefore the alignment procedure could be carried out in a straightforward convenient manner.

At SURF-I the position of the orbital plane could be determined and instruments could be aligned with the plane so that the beam and the optic axis are coincident. The position of the orbital plane was determined by scanning in the vertical direction a grazing incidence monochromator set at a short wavelength (about $200 \AA$ ) and oriented so that its entrance slit was horizontal. The position where the maximum output from the monochromator occurs defines the position of the orbital plane. The monochromator was left in this position and the movable aperture, $A$, was scanned vertically to determine the upper and lower slit occultation positions. Setting the aperture at the average of the upper and lower slit occultation positions aligns its center with the center of the entrance slit. The aperture is set on the orbital plane, the entrance slit of the instrument for calibration is then aligned with the aperture and the optic axis can be aligned with the synchrotron radiation beam by centering the radiation transmitted through the entrance slit on the grating.

Unfortunately, during the course of a day's operation at SURF-I thermal gradients in the magnet produced beam motions of up to one milliradian. Such a motion can produce a large variation in the flux illuminating the optical elements of an instrument. As an example, in figure 7 the variation in output flux from a special torroidal grating monochromator at a wavelength of $188 \AA$ correlates very closely with the temporal variation of the position of the beam illuminating the torroidal grating. Notice that a motion of $0.26 \mathrm{mrad}$ produces a flux variation of 7 percent. A beam motion monitor described in the next section was devised to 


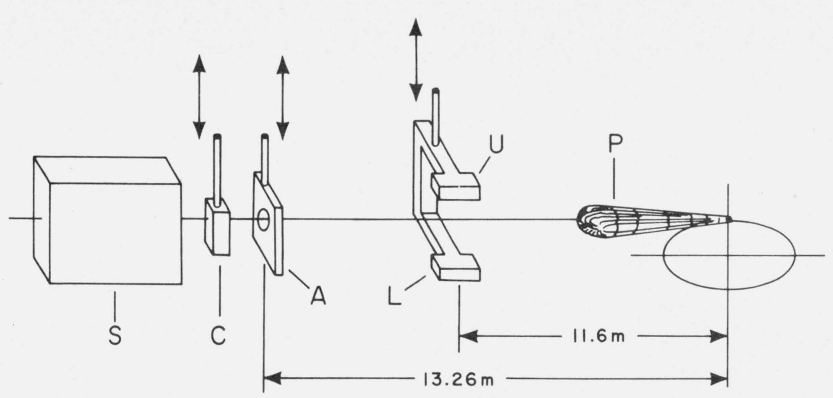

FigURE 6. Schematic diagram of spectrometer calibration apparatus.

Synchrotron radiation $P$, illuminates detectors $U$ and $L$ in the beam motion monitor and the calibrated aperture A. Radiation passing through A illuminates either the spectromete $S$ or the calibrated detector-filter $(C)$

track this beam motion in addition to providing intensity monitoring during calibration. By monitoring the beam motion it was possible to determine that the beam always moved in one direction, that the greatest motion occurred during the first hour or so of operation, and finally that the daily motion of the beam was regular. A calibration could be carried out when the beam was positioned within acceptable limits.

Beam motion was a potential source of systematic error and a needless complication to the calibration procedure. The conversion of the NBS synchrotron into a storage ring will virtually eliminate beam motion because (a) there will be no eddy current heating in the magnet and (b) the only other important source of heat, the power dissipated in the magnet excitation coils, has been eliminated by water cooling the coils. Consequently, spectrometer calibration at SURF-II will be much more accurate and straightforward.

\section{Parameter Measurement and Their Uncertainties}

\subsection{Measurement of the Electron Current and Beam Position}

This basic monitoring system consisted of a detectorfilter combination which was calibrated periodically with an irradiance standard and a pair of detectors used both as a beam motion monitor and as an intensity monitor during the spectrometer calibration when the calibrated detector-filter was removed from the beam. The geometrical layout is shown schematically in figure 6. The beam motion monitor was located in front of the calibration aperture 11.6 meters from the tangent point. The central portion of the beam of radiation passed between the monitor diodes to reach the calibration aperture $(A)$. The beam was then allowed to enter either the calibrated detector-filter $(C)$ or the spectrometer $(S)$. The two diodes serving as the beam motion monitor $(U, L)$ were separated by an angle of 3.3 mrad. Nicol prism polarizers placed in front of these detectors were adjusted to view the component of the beam polarized perpendicular to the orbital plane.

Each detector was displaced symmetrically about the orbital plane and viewed radiation for which the

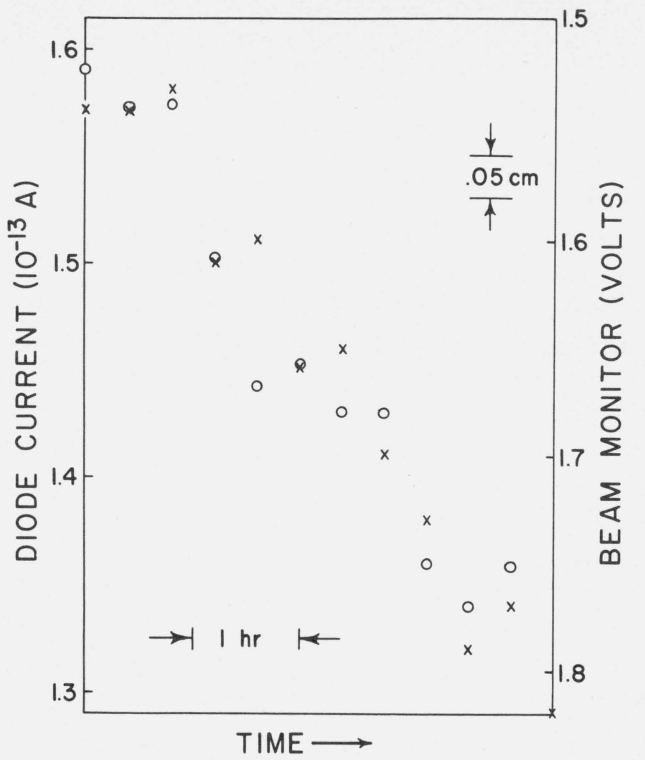

FIGURE 7. Output from the beam motion monitor (BMM) shown as 0 and the output of $a$ diode shown as $\mathrm{x}$ versus time.

The diode measures the vuv flux diffracted by a grating illuminated directly by the synchrotron radiation beam.

intensity varied linearly with angle. This is illustrated in fig. 8. Thus if the beam moved upward slightly the signal in the upper detector $(U)$ would decrease an amount proportional to the beam motion and the signal in the lower detector $(L)$ would increase by about the same amount (assuming the sensitivity of the detectors

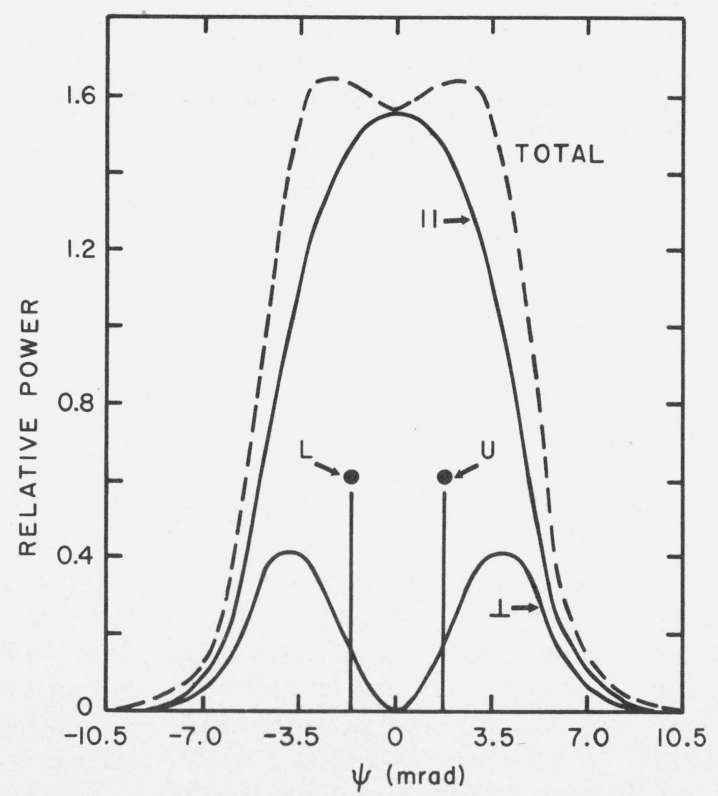

FIGURE 8. Angular distribution of the synchrotron radiation show. ing the total angular distribution of the flux as well as the com. ponent of the flux polarized parallel $(\|)$ and perpendicular $(\perp)$ to the orbital plane.

The angular position of the upper $(U)$ and lower $(L)$ beam motion monitors is shown. 
are the same). Thus the beam motion, $\Delta \phi$, is proportional to the fractional change of the signal level in the detectors. To first order the beam displacement $\Delta \phi=K(U-L) /(U+L)$, assuming that the sensitivity of the detectors is adjusted equal. The output from the upper detector $(U)$ and the lower detector $(L)$ were amplified and manipulated so that the sum $(L+U)$ and difference $(L-U)$ were obtained. Using the $(L-U)$ signal as the numerator input and the $(L+U)$ signal as the denominator input to a quarter-square analogue dividing circuit, the output ratio $R=\frac{L-U}{L+U}$ was proportional to the beam position. A block diagram of the electronic circuitry of the beam motion is shown in fig. 9a. The monitor was calibrated by moving the

a)

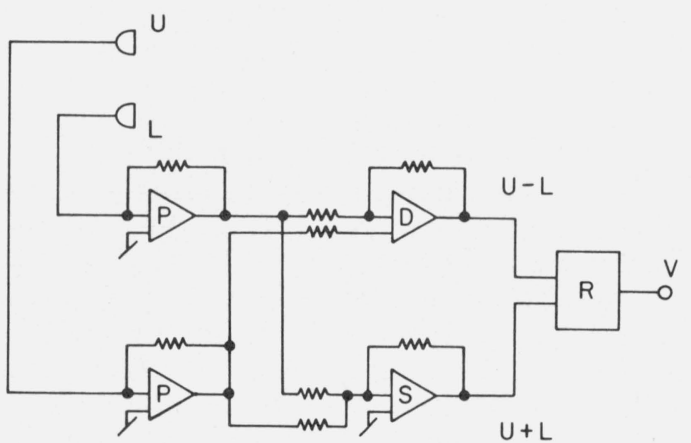

b)

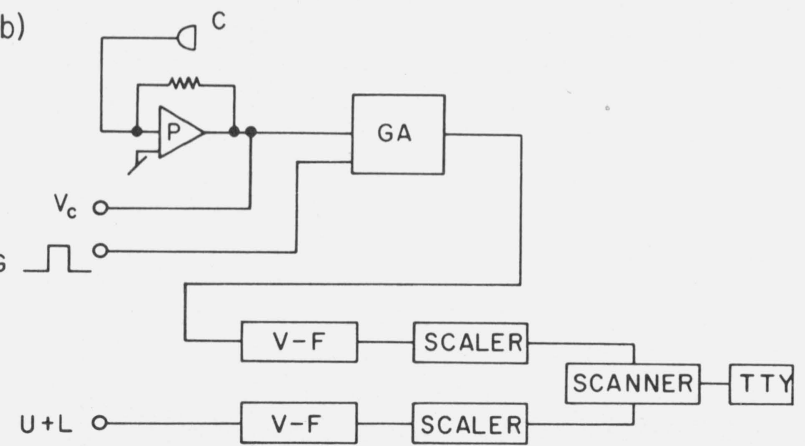

FIGURE 9. (a) Schematic of the electronic circuitry of the beam motion monitor.

Photocurrents from $U$ and $L$ are converted to voltages by $P$. The sum and difference of these voltages are generated in the amplifiers $S$ and $D$ respectively. The output of $S$ and $D$ is the input for a quarter square divider $R$, which provides the ratio $(U-L) /(U+L)$.

(b) Schematic of the electronic circuitry used to transfer calibration from calibrated detector to $\mathrm{U}+\mathrm{L}$.

Photocurrent from calibrated detector-filter combination $C$ is converted to a voltage by $P$. The output of $P$ or a calibration $V_{e}$ is the input of a gated amplifier GA whose output is digitized along with $U+L$, the sum signal from the beam motion monitor. The signals $U+L$ and GA are counted on scalers, scanned and printed on a teletype.

detectors some distance and noting the signal change in $R$. The calibration curve is shown in fig. 10. The sum signal $(L+U)$ to first order is independent of beam position and was used as an intensity monitor during actual spectrometer calibrations to determine the temporal variation in $n_{e}$ while $R$ monitored the change

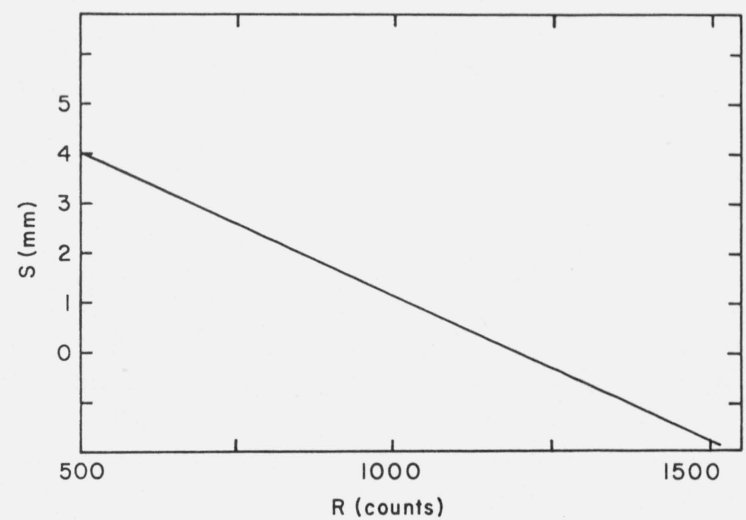

FIGURE 10. The position (S) of the beam motion monitor (BMM) versus the output of ratio $\mathrm{R}$ of the BMM.

in beam position. The calibrated detector-filter system provided the absolute measurement of $n_{e}$.

An improved system for locating the orbital plane will be used at SURF-II. A pair of open xuv diodes covered with thin carbon foil filters (transmitting between 40 and $250 \AA$ ) will be placed above and below the orbital plane. The short wavelength is used to minimize the divergence of the synchrotron radiation beam (see fig. 2) so as to obtain maximum sensitivity. The two diodes will be mounted on a rotatable plate allowing their positions to be interchanged. Thus the position of the orbital plane can be determined as the height at which an interchange of the diodes produces no change in the ratio of the signals from the diodes. This system is expected to be a big improvement over that used on SURF-I.

The temporal stability of the sensitivity of the calibrated detector-filter system and the monitor detectors was sufficient so that it was adequate to calibrate the detector-filter system with an irradiance standard just before and just after the actual spectrometer calibration took place. In a similar manner the calibration was transferred to the monitor diodes sometime during the calibration. Figure $9 \mathrm{~b}$ shows schematically the measuring system used with the calibrated detectorfilter system. The instantaneous voltage is measured by putting the output of the photodiode amplifier (which is fast enough to track the time variation of the light during the acceleration cycles) into a linear gate that is synchronized to the repetition frequency of the accelerator. A peak-reading voltmeter is used to measure and suitably average the voltage pulses that are proportional to $n_{e}$. The width and position of the gate input to the gated linear amplifier is suitably chosen so that the light output in the visible is approximately constant over that portion of the acceleration interval. For the accelerated electrons the complicated integral over the time variation of the electron energy is replaced by a small correction factor amounting to a few percent of the value of flux obtained for monoenergetic electrons of energy $E_{\max }$. This numeric simplification is offset to some extent by more complicated electronics. For stored monoenergetic electrons it is unnecessary to use a gated amplifier, an ordinary 
digital voltmeter or other suitable recording device can be used to measure the voltage and, moreover, no correction is necessary because the electron energy is constant. Because of these factors, $n_{e}$ can be determined much more accurately for stored electrons than for accelerated electrons.

Two types of spectrometers have been calibrated at SURF-I a grazing-incidence monochromator [26] and a Wadsworth [27] spectrometer using photographic detection. For the monochromator it was easy to use the same visible detector gate for gating the output of the monochromator. For the spectrograph, $n_{e}$ was assumed constant over the acceleration cycle. This assumption was tested and will be discussed in the section 4.3.

The NBS irradiance standard used to calibrate the filter-detector system was too intense to be used at the calibration distance (1 meter). To achieve the signal level appropriate to the synchrotron $(\mathrm{nW} / \mathrm{nm})$, the calibration was carried out with the lamp located at distances between $25 \mathrm{~m}$ and $40 \mathrm{~m}$. Thus the validity of the inverse square law was assumed in order to scale the intensity from the lamp calibration distance $(1 \mathrm{~m})$ to the detector calibration distance $(25 \mathrm{~m}-40 \mathrm{~m})$. We determined that the measurements over the $25 \mathrm{~m}^{-}$ $40 \mathrm{~m}$ path were consistent with the inverse square law to \pm 1.0 percent, the uncertainty level of the measurements. Reproducibility of the measurements with respect to the realignment, lamp current adjustment, detector orientation, etc. was \pm 2 percent. Lamp calibration was tested by comparing the working irradiance standard with another standard. The intensity of the working standard derived from the comparison deviated less than 2 percent from its specified value. Combining these sources of errors in quadrature, the quantity $S\left(\lambda_{0}\right)$ in eq 12 could be determined with a probable error of 3 percent. In table 2 we have listed the sources of the errors that contribute to the uncertainty of $n_{e}$. Combining all these errors in quadrature we find that $n_{e}$ can be determined with a probable error of 6 percent.

Possible sources of systematic errors that pertain

TABLE 2. Sources of errors contributing to the uncertainty in the determination of the number of electrons, $\mathrm{n}_{\mathrm{e}}$, in $S U R F-I$.

\begin{tabular}{l|c}
\hline \multicolumn{1}{c|}{ Source of Error } & Magnitude of error \\
\hline $\begin{array}{l}\text { Transfer of calibration to beam motion } \\
\text { monitor }\end{array}$ & $3 \%$ \\
$\begin{array}{l}\text { Determination of change in detector effi- } \\
\text { ciency }\end{array}$ & $3 \%$ \\
Correction factor for accelerated electrons & $1 \%$ \\
$S\left(\lambda_{0}\right)$ & $3 \%$ \\
$C$ & $1 \%$ \\
$P\left(E_{\max }, \lambda_{0}\right)$ & $1 \%$ \\
$V_{F}$ & $1 \%$ \\
$V_{S}$ & $1 \%$ \\
&
\end{tabular}

to the determination of $n_{e}$ were investigated such as the assumption that the standard lamp was a point source (implying the validity of the inverse square law), the possibility that the detector interference filter was not properly blocked, or the presence of reflected radiation sources either at the synchrotron or the lamp calibration hall. From an analysis of these possible sources of errors and other tests, we estimate that sources of systematic errors might contribute a 5 percent uncertainty to the measurement of $n_{e}$.

A storage ring (SURF-II) is now the source of synchrotron radiation at NBS. Using improved techniques and fewer transfers with SURF-II, the uncertainty in the measurement of $n_{e}$ should be reduced by at least a factor of two to a probable error of 3 percent. By further testing and cross checking we expect to reduce the systematic error by the same amount. It is very clear that the increased stability both in time and in space achieved at SURF-II will result in an excellent calibration facility for wavelengths in the vuv. Furthermore with the low intensity electron counting method mentioned section 3.2 the error is zero since the total number of electrons is known exactly.

The method of positioning the spectrometer at SURF-I was described in the early paragraphs of this section. The estimated uncertainty in positioning the instrument on the orbital plane according to that method is $\pm 3 \mathrm{~mm}$ at a distance $13.26 \mathrm{~m}$ from the tangent point or \pm 0.2 mrad. Beam motion monitor drift and other uncertainties associated with the monitor system would increase the positioning uncertainty to $\pm 4 \mathrm{~mm}$ or $\pm 0.3 \mathrm{mrad}$. To estimate the error caused by misaligning a spectrometer with respect to the orbital plane, the flux that passed through a circular aperture $1 \mathrm{~cm}$ in diameter, located a specified angle off the orbital plane, was calculated. The fractional error in the flux determination for such a misalignment is shows as a function of wavelength in fig. 11. As expected the greater the misalignment the greater the error. However, for displacements of up to $0.4 \mathrm{mrad}$ the error is less than 10 percent for wavelengths greater than $200 \AA$. For misalignments of $0.8 \mathrm{mrad}$ the error exceeds 10 percent only for wavelengths less than $600 \AA$. This strong dependence of the error on wavelength is due, of course, to the highly collimated nature of synchrotron radiation, particularly the fact that short wavelength radiation is more tightly collimated than longer wavelength radiation (see fig. 2). Therefore, as the aperture is displaced with respect to the orbital plane, the shorter wavelength radiation becomes occulted first while the longer wavelength radiation, due to its broader angular distribution, is relatively unimpeded. By confining the alignment error to $\pm 4 \mathrm{~mm}$, $(0.3 \mathrm{mrad})$ at $\mathrm{SURF}-\mathrm{I}$ the fractional error in intensity is limited to something less than 5 percent at $200 \AA$ decreasing to about 0.5 percent at $1200 \AA$.

At the SURF-II calibration facility the position of orbital plane will be known to high precision by means of the orbital plane locator described in section 4.1. Therefore errors introduced bacause of uncertainties in the beam position will be negligibly small. 


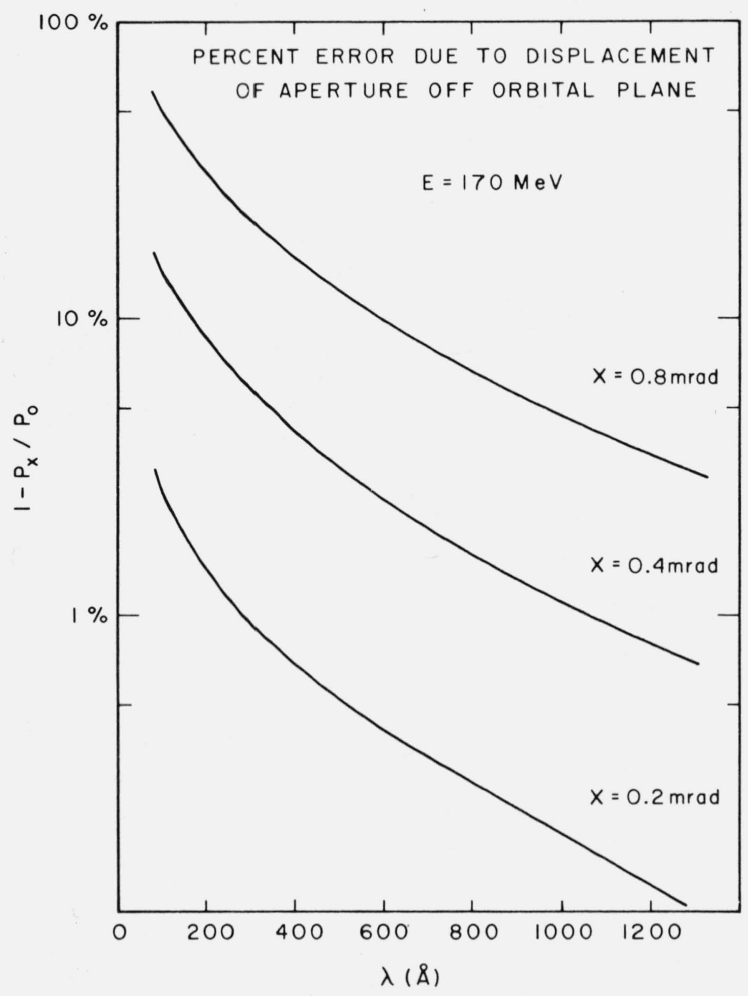

FigURE 11. The percent error due to the displacement of aperture $A$ in figure 7 by the indicated angle $\mathrm{X}$ from the orbital plane.

The curves were calculated for accelerated electrons whose maximum energy was $170 \mathrm{MeV}$

\subsection{Uncertainty in the Orbital Radius of the Electrons}

The magnet geometry at SURF-I and SURF-II is such that the electrons travel in very nearly circular orbits. Thus $R$, the orbital radius, is simply related to the rf accelerating frequency $\nu_{0}$ (known to a few partsper-million). However in practice, since the beam executes small betatron oscillations about the equilibrium orbit, knowledge of the value of $R$ is limited by the extent of these horizontal oscillations which typically have an radial amplitude of a few mm. However since the oscillations result in an instantaneous radius that is equally likely to be larger or smaller than the equilibrium radius, errors in flux determinations tend to cancel. The beam also executes betatron oscillations in the vertical plane which smear the angular distribution somewhat. The extent of this smearing has been computed [28] and while the sum of the intensity due to both polarizations changes very little from the distribution of radiation by a point source of electrons, the magnitude of individual polarization components is affected somewhat. The effect is very small in SURF-I because the observations [4] of both polarization components deviated only slightly from distribution expected for a point source. For the spectrometer calibrations achieved on SURF-I a point source was assumed and the flux distribution was computed accordingly. We estimate that this assumption contributes an error of less than 1 percent to the total flux calculations. Also the beam rather than being 100 percent polarized in the orbital plane is about 95 percent polarized. The response of a spectrometer to both planes of polarization while an important test does not affect the accuracy of the instrument calibration for unpolarized radiation since the average response to both orientations is used. However to properly determine the amount of polarization an instrument introduces to unpolarized radiation the degree of polarization of the radiation on the instrument should be measured.

\subsection{Determination of $E$, the Electron Energy}

In its early life the synchrotron supplying radiation for SURF-I was used for the production of gamma rays. The maximum energy of the electrons accelerated in this synchrotron was determined, at that time, both from the threshold energy for the production of $\pi^{0}$ mesons and from the kinematics of the reaction $h \nu+\mathrm{C}^{12} \rightarrow p+\mathrm{B}^{11}$. An integrating fluxmeter measured the ac magnetic field at a point away from the electron orbit, to which the energy calibration of the nuclear reaction could be attached (since $E_{\text {max }} \propto$ $\left.B_{\max }\right)$. This fluxmeter was also used to measure the maximum electron energy when the synchrotron became the radiation source for SURF-I. At the time of calibration the maximum energy of the electrons was known to 0.6 percent.

At SURF-I measurements were made to test the assumption made in section 3.1 that (a) the electron energy varied sinusoidally and (b) that the number of electrons captured, $n_{e}$, was constant over the acceleration cycle. A grazing incidence monochromator was set at $212 \mathrm{~A}$. The output pulses from this monochromator were accumulated in a multichannel analyzer (MCA) whose sweep was synchronized with the acceleration cycle of the synchrotron. The analyzer display, shown as open circles in fig. 12, represents the instantaneous intensity (averaged over many machine cycles) as a function of time. The dwell time for each channel is $50 \mu \mathrm{s}$. The vertical aperture of the apparatus to the $212 \AA$ radiation was large enough so that all of the radiation entered the monochromator. The solid line represents the flux integrated over all azimuthal angles and normalized to the peak of the time distribution. The flux was calculated for an electron energy variation proportional to $\sin (2 \pi 60 t)$. The time scale has an arbitrary zero so that the maximum count rate, which corresponded to the maximum electron energy, occurred at $t=2.2 \mathrm{~ms}$ rather than $t=4.17 \mathrm{~ms}$. Note the step at $3.6 \mathrm{~ms}$, about $1.4 \mathrm{~ms}$ after the maximum count rate. The rf voltage is turned off at this time because the electrons can no longer be maintained in a stable orbit as the magnetic field decreases. From a comparison of the calculated curve with the observations it is quite plain that the electron energy is proportional to $\sin 2 \pi 60 t$. If the number of electrons captured per cycle were not constant then the data in fig. 12 would not fit the symmetrical calculated curve. As a by-product of the measurement we may note that 


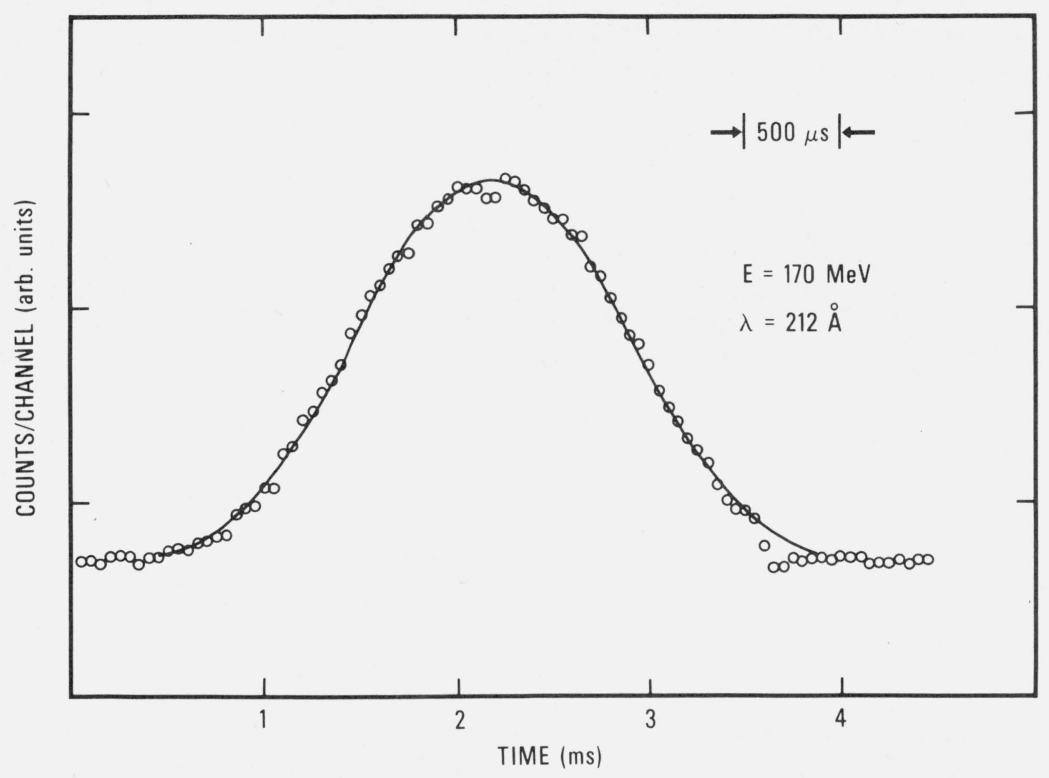

Figure 12. Photon flux per $50 \mu$ s channel dwell time versus time (zero displaced) shown as 0 .

The flux was integrated over many cycles at $212 \AA$ for electrons accelerated to a maximum energy of $170 \mathrm{MeV}$. The solid line is the calculated photon flux.

if the maximum energy were much greater than 170 $\mathrm{MeV}$, the full width at half maximum of the distribution would be somewhat wider since the electrons would radiate at $212 \AA$ sooner, broadening the time distribution. However, this is not a very sensitive electron energy indicator because for an electron whose peak energy is $170 \mathrm{MeV}$ a $10-\mathrm{MeV}$ variation in energy produces only a 7 percent variation in the full width at half maximum of the time varying light flux at $200 \AA$. Nonetheless, the observations are consistent with a maximum electron energy of $170 \mathrm{MeV}$ with an uncertainty of $\pm 5 \mathrm{MeV}$. As a further consistency check the accuracy of maximum electron energy changes was tested by another experiment. As before the output of the monochromator at $212 \AA$ was recorded by a MCA synchronized to the acceleration cycle of the synchrotron. The maximum electron energy was first set at $170 \mathrm{MeV}$ and the signal was integrated for a time determined by integrating the light flux in the visible to some predetermined level. The maximum energy was then set at $160 \mathrm{MeV}$ and the integration was carried out using the multichannel analyzer until the visible light integration reached the same level as before. Since the visible flux is very nearly independent of energy (the flux at $5150 \AA$ for $160 \mathrm{MeV}$ electrons is only about 2 percent less than the flux at $5150 \AA$ for $170 \mathrm{MeV}$ electrons), an equal number of electrons contribute to the time distribution for $E_{\max }=170 \mathrm{MeV}$ as for $E_{\max }=160 \mathrm{MeV}$ (once the 2 percent correction is made). As these curves are normalized to the same number of electrons, the instantaneous flux radiated by electrons when their energy is $E$ should be the same independent of the eventual maximum energy. If $E_{\max }=170 \mathrm{MeV}$ the flux radiated by these electrons at
$160 \mathrm{MeV}$ should equal the flux radiated by electrons at their maximum energy when $E_{\max }=160 \mathrm{MeV}$.

In figure 13 the $170 \mathrm{MeV}$ data is shown as dots while the $160 \mathrm{MeV}$ data (normalized to the same total number of electrons) is shown as solid squares. The large tic on the abscissa indicates the time when electrons whose maximum energy is $170 \mathrm{MeV}$ have an instantaneous energy of $160 \mathrm{MeV}$. A horizontal line indicating the instantaneous flux radiated by $160 \mathrm{MeV}$ electrons guides the eye to the data where the maximum electron energy is $160 \mathrm{MeV}$. The correspondence between the data is quite clear. Two short horizontal lines, one above and the other below the data for which the maximum electron energy was $160 \mathrm{MeV}$, indicate the instantaneous flux radiated by electrons of energy $158 \mathrm{MeV}$ and $162 \mathrm{MeV}$. From figure 13 we see a change of electron energy of $2 \mathrm{MeV}$ produces a rather large change in intensity at this wavelength. From this data we infer that the probable error in the energy change of $10 \mathrm{MeV}$ was about $0.2 \mathrm{MeV}$.

To determine the systematic error introduced by the quoted uncertainty in the maximum energy $(0.6 \%)$, the percent change in flux for an electron energy change of $1 \mathrm{MeV}(0.6 \%)$ at $170 \mathrm{MeV}$ is plotted as a function of wavelength in figure 14. Notice as the wavelength increases the photon flux change is much less sensitive to changes in the electron energy. At $200 \AA$ such an uncertainty in energy results in a 9 percent uncertainty in the photon flux. This uncertainty decreases monotonically to 1 percent at a wavelength of approximately $2000 \AA$.

At the SURF-II storage ring a great deal of effort has been expended to measure the (now dc) magnetic field at the orbit to 0.1 percent. A highly accurate 


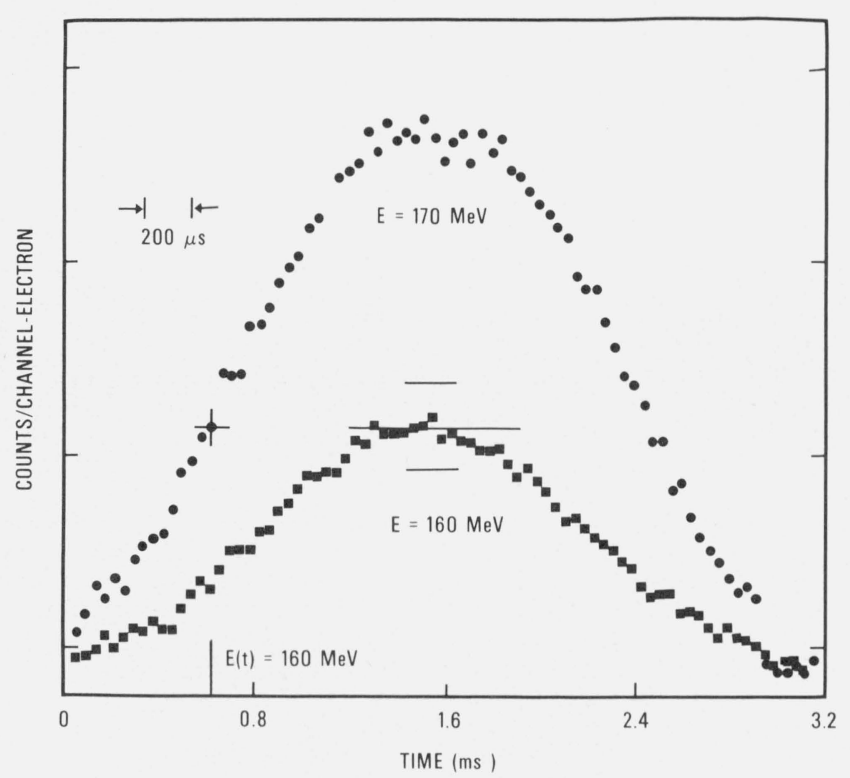

FIGURE 13. Instantaneous photon flux normalized to the same total beam current for two maximum energies: $\mathrm{E}_{\max }=170 \mathrm{MeV}$ and $\mathrm{E}_{\max }=160 \mathrm{MeV}$.

Vertical line on abscissa denotes time when electrons with $E_{\max }=170 \mathrm{MeV}$ have an energy of $160 \mathrm{MeV}$. The horizontal lines indicate the maximum flux expected if $E_{\max }=162$ $\mathrm{MeV}$ or if $E_{\max }=158 \mathrm{MeV}$.

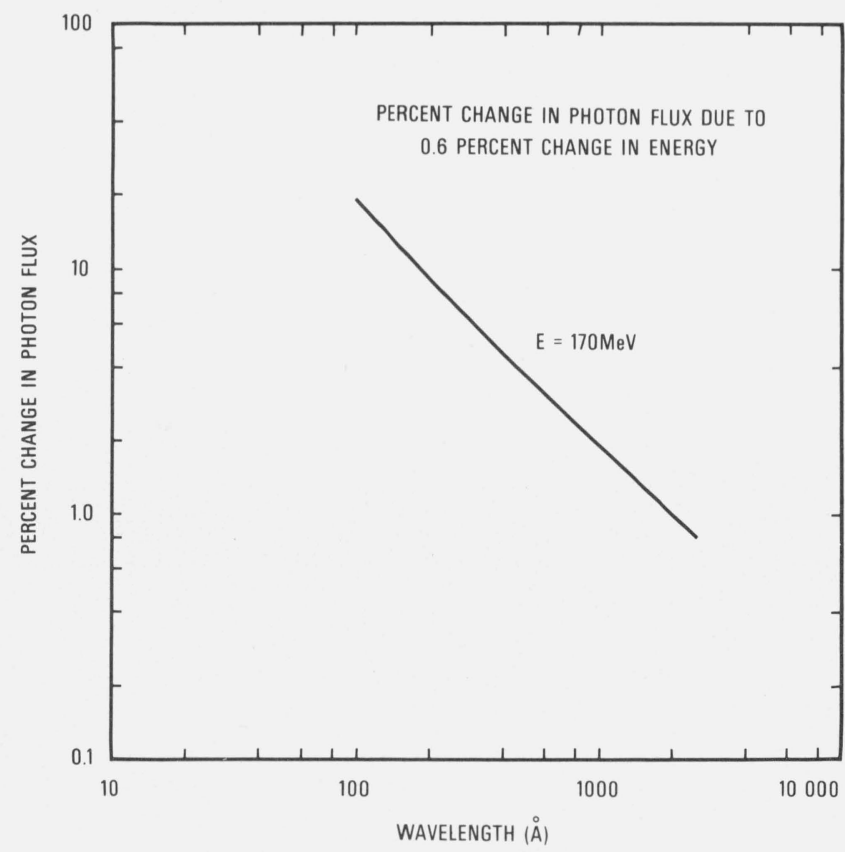

Figure 14. Percent change in photon flux emitted by accelerated electrons with $\mathrm{E}_{\max }=170 \mathrm{MeV}$ due to a 0.6 percent change in electron energy.

Hall probe (calibrated by the NBS magnetic field calibration group to $0.1 \%$ ) is inserted at a point on the electron orbit. Because an active probe would interfere with the electron beam, the orbital field calibration was transferred to the Hall probe field monitor located away from the electron orbit.

With the magnetic field and hence the electron energy known to this accuracy, radiometric uncertainties will be much less, as is indicated in figure 15. In this figure we show as a function of wavelength the flux change, passing through an aperture subtending an angle 2 mrad centered on the orbital plane and $1 \mathrm{mrad}$ along the orbit, that is due to a change in electron energy of 0.1 percent. The flux change for $200 \mathrm{MeV}$ monoenergetic electrons is shown in the top curve while the bottom curve represents the flux change for $240 \mathrm{MeV}$ monoenergetic electrons. With the electron energy known to this accuracy, it is possible to keep the uncertainties in photon flux less than 5 percent for all wavelengths greater than $40 \AA$, the practical short wavelength limit of our storage ring. Now instead of being one of the major sources of systematic error, especially at shorter wavelengths, the uncertainty in the energy introduces one of the smaller errors into the calibration process.

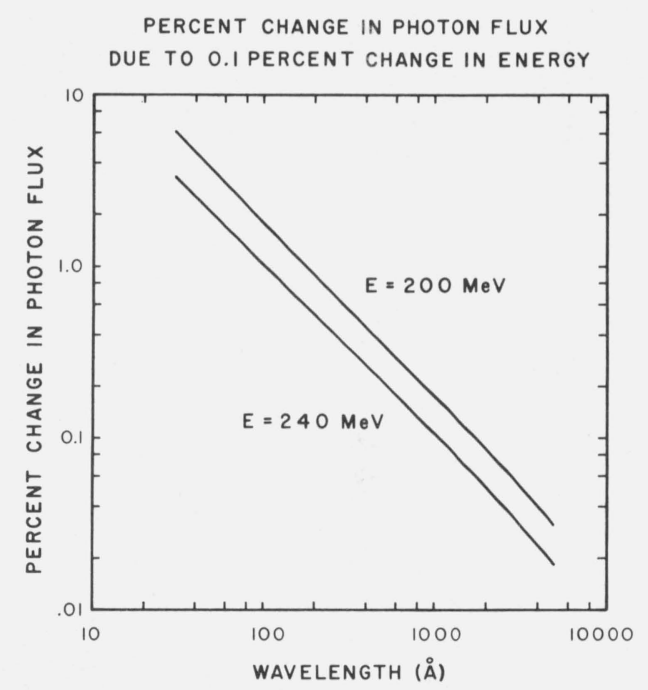

FigURE 15. Percent change in photon flux emitted by monoenergetic electrons with the energies shown for a 0.1 percent charge in the electron energy.

The uncertainty of 0.1 percent is that expected at SURF-II.

\section{Summary}

In this report we have dealt with the use of synchrotron radiation as an absolute irradiance standard in the vacuum ultraviolet in general. In addition, we detailed the calibration procedures devised at the NBSSURF-I facility for several spectrometer calibrations. General sources of systematic errors were identified and their importance was studied relative to SURF-I. Improved calibrations are anticipated at the NBS storage ring (SURF-II) based mainly on better energy determination of the monoenergetic electrons, higher spatial and temporal beam stability due to the storage ring operation and finally, a more accurate determina- 
tion of the circulating current. Table 3 is a final summary of the sources of errors for SURF-I and SURF-II and the role they play in the overall accuracy of a calibration.

TABLE 3. Sources of errors in the use of synchrotron radiation as an absolute source

\begin{tabular}{l|l|c|c}
\hline \hline & Type $^{\mathrm{a}}$ & \multicolumn{2}{|c}{ Magnitude of error } \\
\hline \multicolumn{1}{c|}{ Error source } & & SURF-I & SURF-II \\
\hline Determination of $n_{e}$ & $R$ & $6 \%$ & $3 \%$ \\
& $S$ & $6 \%$ & $3 \%$ \\
Electron energy calibration & $S$ & $8 \%-1 \%$ & $5 \%-0.04 \%^{\mathrm{c}}$ \\
Electron energy variation & $R$ & $8 \%-1 \%{ }^{\mathrm{b}}$ & Negligible \\
Orbital radius & $S$ & $<2 \%$ & $<1 \%$ \\
Uncertainty in beam & $S$ & $5 \%-0.5 \%$ & $<1 \%$ \\
position & $S$ & $<1 \%$ & $<1 \%$ \\
Finite source size & $S$ & $<4 \%$ & $<1 \%$ \\
Precision of calculated & $S$ & $11 \%-6 \%$ & $3 \%$ \\
model & $R$ & $<10 \%$ & $<6 \%$ \\
$\quad$ Total & $S$ & \multicolumn{2}{|c}{} \\
\end{tabular}

${ }^{\text {a }} R$ designates random errors and $S$ designates systematic errors.

b The first value is the uncertainty at $200 \AA$ and the second value is the uncertainty at $2000 \AA$.

c The first value is the uncertainty at $40 \AA$ and the second value is the uncertainty at $2000 \AA$.

${ }^{\mathrm{d}} \mathrm{A}$ readout of electron energy will be available.

\section{References}

[1] Canfield, L. R., Johnston, R. G., and Madden, R. P., Appl. Opt. 12, 1611 (1973).

[2] Saloman, E. B., and Ederer, D. L., Appl. Opt. 14, 1029 (1975).
[3] Tomboulian, D. H., and Hartman, P. L., Phys. Rev. 102, 1423 (1956).

[4] Codling, K., and Madden, R. P., J. Appl. Phys. 36, 380 (1965).

[5] Bathow, G., Freytag, E., and Haensel, R., J. Appl. Phys. 37, 3449 (1966).

[6] Lemke, D., and Labs, D., Appl. Opt. 6, 1043 (1967).

[7] Boldt, G., Space Sci. Revs. 11, 728 (1970).

[8] Ott, W. R., Fieffe-Prevost, P., and Wiese, W. L., Appl. Opt. 12, 1618 (1973).

[9] Böhm, W., and Labs, D., Appl. Opt. 10, 2021 (1971).

[10] Van Raan, A. F. J., Physica 65, 566 (1973).

[11] Pitz, E., Appl. Opt. 8, 255 (1969).

[12] Ott, W. R., and Wiese, W. L., Optical Engineering 12, 86 (1973).

[13] Zaidel, A. N., Malyshev, G. M., and Shreider, E. Ya., Zh. Tekh. Fiz. 31, 129 (1961) [Eng. trans. Soviet Phys.-Tech. Phys. 6, 93 (1961)].

[14] Gladushchak, V. I., and Shreider, E. Ya., Opt. i Spektroskopia 14, 815 (1963) [Eng. Trans., Opt. Spectr. 1 7, 75 (1964)].

[15] Mumma, M. J., and Zipf, E. C., J. Opt. Soc. Am. 61, 83 (1971).

[16] Rusbüldt, D., and Thimm, K., Nucl. Instrum. Methods 1 16, 125 (1974).

[17] Godwin, R. P., Springer Tracts in Modern Physics Vol. 51 (Berlin: Springer-Verlag (1969)).

[18] Codling, K., Rep. Prog. Phys. 36, 541 (1973).

[19] Madden, R. P., Synchrotron Radiation and Applications, Chapter in X-Ray Spectroscopy, Ed., L. Azaroff (McGrawHill, 1974) pp. 338-378.

[20] Key, P. J., Metrologia 6, 97 (1970).

[21] Ott, W. R., and Bartoe, J. D., J. Opt. Soc. Am. 62, 1372 (1972).

[22] Struck, D., and Wende, B., J. Opt. Soc. Am. 62, 96 (1972).

[23] Schwinger, J., Phys. Rev. 75, 1912 (1949).

[24] Saloman, E. B., Appl. Opt. 14, 1391 (1975).

[25] Fairchild, T., private communication.

[26] Higgins, James, Salter, R., and Heroux, Leon, private communication.

[27] Bartoe, John, and Bruckner, Keith, private communication.

[28] Zhukovskii, V. Ch., and Shishanin, O. E., Opt. i Spektroskopia 31, 857 (1971) [Eng. Trans., Opt. Spectr. 31, 465 (1971)].

(Paper 79A6-873) 